\section{Cahiers de Narratologie}

Analyse et théorie narratives

10.2 | 2001

La voix narrative

\title{
Ambiguïtés narratives et ironie dans Hôtel du lac d'Anita Brookner
}

\section{Eileen Wanquet}

\section{Q OpenEdition}

1 Journals

\section{Édition électronique}

URL : http://journals.openedition.org/narratologie/10314

DOI : 10.4000/narratologie.10314

ISSN : 1765-307X

Éditeur

LIRCES

\section{Édition imprimée}

Date de publication : 1 janvier 2001

Pagination : 525-537

ISBN : 2914561032

ISSN : $0993-8516$

\section{Référence électronique}

Eileen Wanquet, «Ambiguïtés narratives et ironie dans Hôtel du lac d'Anita Brookner », Cahiers de Narratologie [En ligne], 10.2 | 2001, mis en ligne le 01 janvier 2001, consulté le 11 juin 2020. URL:

http://journals.openedition.org/narratologie/10314; DOI : https://doi.org/10.4000/narratologie.10314 


\title{
AMBIGUÏTÉS NARRATIVES ET IRONIE DANS HOTEL DU LAC D'ANITA BROOKNER
}

\author{
Eileen WANQUET \\ Université de La Réunion
}

Anita Brookner, romancière contemporaine britannique, née en 1928, a aujourd'hui soixante-douze ans et vit à Londres. Cette historienne de l'art a commencé à écrire des romans à l'âge de cinquante-trois ans. Depuis, elle en a publié dix-neuf, à raison d'un roman par an.

Nous nous proposons de faire porter notre attention sur Hotel du Lac, quatrième roman de Brookner qui lui valut le Booker Prize en 19841. L'approche adoptée est celle de la narratologie "classique ", fondée sur les travaux de Gérard Genette, en tenant compte des mises au point faites par Miecke Bal et Pierre Vitoux ${ }^{2}$. Notre analyse utilise aussi l'approche complémentaire que Dorrit Cohn expose dans $L a$ Transparence intérieure ${ }^{3}$. Ces outils théoriques nous permettront une analyse détaillée du texte, qui aidera d'une part à mieux cerner l'origine du sentiment de malaise qui semble se dégager dès la première page, d'autre part à interpréter la fin ambiguë du roman. Il s'agira de montrer à quel point toute la mise en scène est ironique, car parcourue de dédoublements, de décalages, de tensions.

${ }^{1}$ La traduction utilisée ici est celle de Solange Lecomte, publiée par Belfond en 1988.

2 Voir : Pierre VITOUX, «Le jeu de la focalisation " in Poétique, $\mathrm{n}^{\circ} 29$, février 1977, pp. 359-368.

3 Dorrit COHN, La transparence intérieure: Modes de représentation de la vie psychique dans le roman, (Traduit de l'anglais par Alain Bony), Collection Poétique, Paris, Seuil, 1981. 


\section{I - Voix narratives, modes de discours et focalisation}

La description initiale du lieu, les abords du Lac Léman, est en apparence purement référentielle, mais donne néanmoins une impression diffuse de malaise. Tout en étant limitée par le cadre d'une fenêtre, la perception du paysage se situe dans un infini insaisissable : "En regardant par la fenêtre, on ne voyait rien d'autre qu'un terrain qui se perdait dans le gris » (HL 9). Malgré la profusion de détails réalistes, l'atmosphère est irréelle et sans vie dans un univers silencieux. L'utilisation de pronoms indéfinis et la longueur des phrases complexes accentuent l'effet d'endormissement souligné par l'image : "l'immense lac gris dont les eaux s'épandaient, comme on diffuse un anesthésique ». La deuxième partie de la description s'ouvre trompeusement sur "un nouveau paysage, plein de couleur et de mouvement ", mais l'effet de " collage » obtenu par une énumération figée fait que le paysage reste sans vie, telle une carte postale faite de morceaux disparates. Le long paragraphe initial se termine par une antithèse : "Ce pays était, en effet, une terre d'abondance... où l'on savait prévenir les défaillances humaines et où, seul, le désolant climat restait incontrôlable ». Entre ce qui est contrôlable et incontrôlable se glisse une atmosphère d'irréalité, de vide, de silence.

L'impression d'avoir affaire ici à un narrateur focalisateur extradiégétique décrivant un paysage de manière neutre et objective, est remise en cause dès le deuxième paragraphe, qui débute de la manière suivante : "Edith Hope [. . . ] resta un moment debout à sa fenêtre " ( $H L 10)$. Ainsi, le lecteur se trouverait-il installé, dès les premières lignes, dans la pensée du personnage principal et le malaise ressenti serait celui de l'héroöne.

Ce paragraphe initial est suivi de trois lignes de récit toujours pris en charge par le narrateur extradiégétique, mais cette fois le narrateur est aussi le focalisateur, qui voit Edith debout à sa fenêtre, en focalisation externe. Dans ce récit à la troisième personne et au passé simple, le narrateur présente la vie d'Edith dans une perspective télescopique; se dissociant davantage du point de vue de l'héroïne, le narrateur affirme sa propre supériorité en se permettant un jugement de valeur 
sous la forme d'une boutade : "Edith Hope, qui sous un pseudonyme plus aguicheur écrivait des romans sentimentaux, resta un moment debout à la fenêtre, "

Ensuite, avec le même détachement ironique, le narrateur extradiégétique pénètre dans la pensée de l'héroïne, la focalisant de l'intérieur pendant les neuf lignes suivantes introduites par un " comme si » ironique, qui implique un verbe de pensée : "comme si [elle pensait que] elle pouvait encore parvenir, avec de la bonne volonté, à percer la mystérieuse opacité qu'elle affrontait depuis son arrivée...» Le récit narrativisé est ici remplacé par ce que Dorrit Cohn appelle un « monologue [intérieur] narrativisé », en style indirect libre ${ }^{4}$, « le narrateur assumant le discours du personnage, ou si l'on préfère le personnage parlant par la voix du narrateur $»^{5}$. Le narrateur s'est glissé dans la pensée du personnage, et bien qu'il continue à prendre en charge son discours mental il lui cède progressivement la place.

Viennent ensuite treize lignes où l'héroïne se penche sur les événements qui l'ont conduite à son exil à l'hôtel du lac. Il s'agit là de ce que Dorrit Cohn qualifie de « souvenirs narrativisés ${ }^{6}$. Ce retour en arrière, introduit ironiquement par le verbe « oublier », doit avoir recours au plus-que-parfait pour être reconnu comme tel : "qui lui permettrait [...] d'oublier le faux pas lamentable qui l'avait condamnée à s'exiler pour un temps dans cet endroit. . ."

Ensuite, pendant vingt-quatre lignes, nous notons un changement de temps grammatical, du passé au présent, et un changement de personne, de la troisième à la première, nous signalant l'utilisation du discours direct. Les souvenirs narrativisés font place à un "monologue [intérieur] rapporté »7. qu'aucun guillemet ne sépare du contexte à la troisième personne, qu'accompagne une seule formule d'introduction : "Il n'est pas question, bien sûr, qu'on m'absolve; je ne suis

4 Dorrit COHN, op. cit., p. 28.

5 Gérard GENETTE, Figures III, Collection Poétique, Paris, Seuil, 1972, p. 194.

6 Dorrit COHN, op. cit. p. 152.

7 Ibid., p. 26. 
pas une ingénue, pensa-t-elle » (HL10). S'effaçant graduellement, par touches successives, le narrateur a complètement délégué la focalisation à son personnage. $\mathrm{La}$ voix du narrateur n'est plus mêlée à celle du personnage, elle lui a laissé la place. Mais c'est maintenant le personnage qui adopte l'attitude d'un narrateur "omniscient » qui analyse sa vie en psycho-récit, adoptant une perspective télescopique et une sagesse narratriale.

Le mot «maintenant» signale le retour au temps du récit, et introduit un autre changement de focalisation ainsi que de voix narrative. Sortant brusquement des pensées intimes du personnage, nous passons rapidement du monde intérieur au monde extérieur, de la citation au récit à la troisième personne, et du présent au passé. Le focalisateur est à nouveau le narrateur extradiégétique, qui perçoit Edith de l'extérieur, debout à sa fenêtre : «Edith finit par tourner le dos à la morne étendue. .. » (HL 11).

Mais le narrateur délègue aussitôt la focalisation à son héroïne pour la description de la chambre d'hôtel. D'emblée il est cette fois clair que le lieu est décrit du point de vue d'Edith : "et elle se mit à examiner sa chambre, qui avait une couleur de veau trop cuit. .."

Quatorze lignes de récit descriptif font place à deux lignes de monologue rapporté en discours direct: "Quand il fera beau, se dit-elle, je m'y installerai pour écrire"

Viennent ensuite dix lignes de récit : «Avisant alors son sac de voyage, elle alla en retirer deux chemises cartonnées. [...] se mit à écrire », avant l'emploi d'une technique narrative nouvelle : la première des cinq lettres qu'Edith écrit à son amant marié, David.

La lettre, introduite par des guillements, est écrite à la première personne du singulier. Le temps grammatical utilisé est d'abord le passé, pendant les quarante-sept lignes où Edith raconte à David son arrivée à l'hôtel, avant de décrire l'hôtel et ses occupants au présent.

Cette analyse détaillée des cinq premières pages de Hotel du Lac met en lumière la technique narrative utilisée tout au long du roman. Le texte change sans cesse de voix narrative, de point de vue et de mode de discours. 
L'alternance continuelle entre récit, monologue narrativisé et monologue rapporté, l'oscillation entre « je » et « elle », reflète les alternances entre monde extérieur et monde intérieur, ainsi qu'entre la voix du narrateur extradiégétique et celle du personnage, narrateur intradiégétique. Ces alternances de voix sont accompagnées de glissements constants de focalisation : le roman commence sur un ton faussement neutre et objectif, et ne prend que graduellement ancrage dans la conscience du personnage, avant d'effectuer brusquement le mouvement inverse ; autrement dit le focalisateur sujet nondélégué (le narrateur) voit Edith d'abord en focalisation externe, puis en focalisation interne, lui déléguant temporairement la focalisation, pour la reprendre ensuite. La confusion entre la voix du narrateur et celle du personnage, créée par l'utilisation du style indirect libre, est accentuée par le fait que voix narrative et focalisation ne vont pas toujours de pair : parfois le narrateur extradiégétique délègue la focalisation externe de son récit apparemment neutre à son personnage ; inversement, le narrateur-focalisateur intradiégétique adopte parfois le ton d'un narrateur omniscient. L'ambiguïté de la voix narrative est elle-même source de tension, mais l'ironie est accrue par la distance que maintient le narrateur par rapport à son personnage.

De plus, de fréquentes analepses et changements de niveaux narratifs viennent brouiller davantage encore le texte et soulignent l'ironie du regard que porte le narrateur extradiégétique sur son héroïne, envoyée en exil afin «d'oublier son faux pas lamentable » et de «redevenir elle-même».

\section{II - Analepses et niveaux narratifs}

Il ne suffit pas qu'il y ait glissement de focalisation ou de voix narrative pour parler de changement de niveau narratif. Les changements de niveaux narratifs apparaissent toujours clairement situés à l'intérieur de passages pris en charge par le personnage : lorsque les souvenirs de l'héroïne se trouvent inclus dans un monologue narrativisé, l'antériorité temporelle est marquée par un passage du passé simple au plus-queparfait ; lorsque l'héroïne se souvient de son passé dans un monologue rapporté, le présent du discours direct cède la place au passé ; lorsque ses souvenirs sont contés à David 
sous forme épistolaire, l'utilisation de la première personne du singulier est d'emblée associée au passé simple.

Il ne suffit pas non plus qu'il y ait analepse pour parler de changement de niveau narratif. Nous retiendrons ici deux types d'analepses : des " analepses externes », dont «toute l'amplitude reste extérieure à celle du récit premier », constituant ainsi un "récit temporellement second, subordonné au premier », et des « analepses internes homodiégétiques », dont " le champ temporel est compris dans celui du récit premier» et qui portent "sur la même ligne d'action que le récit premier $»^{8}$.

Le récit premier d'Hotel du Lac concerne les deux ou trois mois passés par l'héroïne de trente-neuf ans sur les bords du Lac Léman et couvre environ cent cinquante pages sur un total de deux cents. Le récit second, qui ne couvre qu'une cinquantaine de pages, est constitué par deux séries d'analepses externes, toutes deux portant sur les souvenirs d'Edith : la première série de huit analepses externes reprend dans le désordre les événements significatifs des quatre années ( $H L$ 93) précédant l'arrivée d'Edith à l'hôtel. Ensemble, elles forment une " analepse externe complète ", venant rejoindre le point de départ du récit premier. La deuxième série de quatre analepses externes - "non complètes »- concerne quelques souvenirs d'enfance d'Edith.

Nous avons relevé huit analepses externes (HL 10, 11, $11,12-13,28-33,61-66,91-94,127-144)$ couvrant un total d'environ trente-cinq pages, concernant le passé proche d'Edith. Elles sont toutes prises en charge par l'héroïne, qui s'isole de son entourage - par exemple en fermant les yeux pour se rappeler certains événements marquants de sa vie avant son arrivée à l'hôtel, que ce soit sous forme épistolaire ou en monologue rapporté et surtout narrativisé. Ces souvenirs sont toujours provoqués par un sentiment particulièrement aigu de solitude, de malaise social, de manque, de la

8 Gérard GENETTE, Figures III, op. cit., pp. 90-92. 
part d'Edith ou par la présence d'un homme qui lui fait penser à David: "La récente intrusion, toute parodique qu'elle fût, d'un homme dans le champ de sa conscience, avait eu pour effet de réveiller douloureusement son besoin d'aimer » (HL 60). Mais les souvenirs du personnage sont filtrés par le narrateur, qui leur confère une fonction explicative : les «proches » d'Edith l'ont envoyée à l'étranger pour "redevenir elle-même ", "oublier [son] faux pas lamentable » (HL 10) et «en revenir assagie, mûrie et repentante» ( $H L$ 10). Elle avait accepté d'épouser un célibataire ennuyeux, Geoffrey Long, mais, à la dernière minute, s'était rétractée, choisissant de rester avec son amant marié, David. Ironiquement, loin d'oublier sa 'faute', Edith se réfugie régulièrement dans un passé dont elle est incapable de se libérer et qui l'empêche de vivre pleinement au présent. Alors que le présent du narrateur se situe résolument dans le récit premier, celui de l'héroïne glisse inexorablement vers le passé.

Ces analepses explicatives sont complétées par quatre analepses externes ( $H L$ 51-53, 67, 90, 112), couvrant un total d'environ dix pages et dont le point de portée se situe dans «les brumes de l'enfance » (HL 67) d'Edith, comme si le narrateur voulait faire comprendre au lecteur que les problèmes rencontrés par son personnage ont leurs racines dans un passé plus lointain. Les tristes souvenirs d'enfance d'Edith sont toujours déclenchés par le bonheur et la complicité qu'affichent la charmante Mrs Pusey et sa fille, la pulpeuse Jennifer, car cet "amour entre mère et fille, de contact physique et de complicité dans la coquetterie tout ce qu'elle-même n'avait jamais connu » (HL 51) lui rappelle de douloureux souvenirs de Rosa, sa mère viennoise trop égocentrique et aigrie par la vie pour aimer sa fille "silencieuse " et son mari anglais "devenu peu à peu complètement muet » (HL 90). Le choix de souvenirs que le narrateur attribue à son personnage établit clairement une relation de cause à effet entre le tempérament triste et effacé d'Edith et le manque d'amour maternel de la part d'une mère qui ne lui a pas appris les "règles du jeu», lui léguant seulement «son ignorance et ses illusions » (HL 112). Edith, 
piégée par son passé, n'est pas plus capable de se libérer de sa mère que de son amant.

Le dédoublement du narrateur et du temps du récit est accompagné d'un dédoublement de la personnalité de l'héroïne.

\section{III - Révisions textuelles, dualités et revirements ironiques}

L'utilisation associée d'analepses internes homodiégétiques permet au narrateur de mettre l'accent sur les fausses notes de la subjectivité de son héroïne, qui a hérité des illusions de sa mère. Edith se méprend sans cesse, sur les autres personnages, sur elle-même et sur la conduite à tenir pour atteindre le bonheur. L'héroïne revient constamment sur ses premières impressions et s'auto-critique dans des " analepses internes homodiégétiques répétitives » où «le récit revient ouvertement sur ses propres traces ${ }^{9}$. ce qui n'empêche pas qu'elle se méprenne à nouveau. Si le personnage se permet de réviser constamment le texte, comme le ferait un narrateur omniscient, le narrateur extradiégétique ironique ne se permet jamais de corriger son personnage. Il se contente de placer son héroïne 'aveugle' dans des situations ironiques - il laisse sa victime se tromper jusqu'à ce que l'évidence de son erreur s'impose à elle, soit sous forme d'événements narratifs, soit par le biais des révélations et des opinions des autres personnages. L'ironisant est bien ici le narrateur et l'ironisé, la cible, son héroïne.

Edith, qui se croit très intelligente et porte un regard critique sur les autres, se méprend pourtant sur tous les personnages qui l'entourent à l'hôtel, par exemple sur leur âge, leur statut social ou leur profession. Edith rectifie ellemême ses premières impressions à plusieurs reprises, révise son propre texte, remettant aussitôt en cause chaque hypothèse, mais elle se fourvoie toujours, et ne découvre la vérité que lorsqu'elle lui est révélée par les événements narratifs ou par les autres personnages.

${ }^{9}$ Gérard GENETTE, op. cit., p. 95. 
Pour souligner l'ironie du regard qu'il porte sur son hérö̈ne, le narrateur exploite la vision qu'en ont les autres personnages. L'héroïne croit brouiller les cartes en jouant un rôle, mais son entourage n'est pas si naîf. Edith se conforme, à s'y méprendre elle-même, à l'image que les autres ont d'elle.

Mais les événements narratifs s'associent aux points de vue des autres personnages, pour révéler que celle qui se veut raisonnablement gentille, modeste, sérieuse, douce, serviable, fidèle, en somme éminemment vertueuse, agit en fait comme un monstre d'égoïsme. Elle "s'enorgueillit» de ne faire souffrir personne en étant la maîtresse de David et de ne jamais se plaindre de «ses dimanches vides » (HL 65). Dans son égocentrisme, elle se pose en victime, et ne pense jamais à l'épouse de David. Elle avait décidé d'épouser l'ennuyeux Geoffrey Long, observant froidement qu'il était «la meilleure garantie d'un avenir tranquille et sage " ( $H L$ 127). Puis, à la dernière minute, elle avait ordonné au taxi qui l'emmenait au Bureau des Mariages de continuer sa route, devant les "bouches bées " et les "yeux horrifiés 》 de son fiancé et de ses amis $(H L 139)$. Elle était ensuite rentrée calmement chez elle sans le moindre remords, méprisant totalement tous ses amis. Elle avoue par la suite que «cette chose effarante ne [l'avait] guère préoccupée sur le moment" (HL 11).

Effectivement, Edith a une double personnalité, celle du jour et celle de la nuit. L'autre facette de sa personnalité se révèle la nuit. Le narrateur nous dit que son sommeil est «troublé » (HL 70), ses nuits sont «agitées » (HL 70) par des "rêves incohérents" (HL 69): "Et là, en pleine nuit, son coeur se mit à battre de façon désordonnée tandis que sa raison, cessant de jouer son rôle de censeur, se fragmentait et que, dans sa conscience, des zones d'ombre s'éclairaient brusquement pour révéler de dangereux écueils " (HL 125). Sous la carapace qu'Edith présente au monde, se cache un univers fait de tristesse, d'agitation et d'insatisfaction. Le texte d'Hotel du Lac est parcouru d'images et de leitmotive obsédants qui confirment le sentiment de malaise suscité par le paragraphe initial.

L'ironie avec laquelle elle considère son entourage se retourne doublement contre Edith : non seulement celle qui 
choisit d'offrir à autrui une fausse apparence est démasquée, mais de surcroît elle se méprend sur la vie. M. Neville a compris qu'elle se mentait à elle-même : "ce à quoi vous voudriez croire vous induit en erreur» (HL 102). Monica, qu'Edith prend aussi pour une femme inintelligente, se révèle perspicace : "Et je croyais que vous étiez plus ou moins un écrivain. Un écrivain n'est-il pas en principe un observateur avisé de la nature humaine ou de je ne sais trop quoi? Si je vous le demande, c'est que, parfois, vous m'étonnez et vous me semblez avoir le cuir un peu épais » (HL 155). Edith s'incline humblement : "Ce que je vois est si différent de ce que je m'attends à voir que je n'ai plus confiance en mon propre jugement » (HL 155).

Effectivement, Edith est déchirée entre rêve et réalité, illusions et lucidité, passion et raison, dichotomies qui soustendent toute l'œuvre de Brookner. Edith elle-même distingue bien la différence entre, d'une part l'amour impossible des romantiques et, d'autre part, son aspiration à une vie quotidienne paisible: "On m'a qualifiée de romantique presque toute ma vie. Mais je n'en suis pas une. Je suis un animal domestique. Je n'aspire pas le moins du monde aux débordements passionnels [...] Non, ce dont j'ai grand besoin, c'est de simple routine " (HL 105). Edith pense que c'est sa mère qui lui a légué ses illusions, que M. Neville qualifie de "romantiques». Sa mère "se consolait de ses éternels déboires en lisant des histoires d'amour, des histoires d'amour qui finissaient toujours bien » (HL 112) et Edith constate que, si elle est devenue auteur de romans sentimentaux c'est à cause de sa mère : "Peut-être est-ce pour ça que j'en écris, moi ».

L'illusion d'Edith, dont le nom est ironiquement «Hope »- espoir - est de vouloir concilier amour passion et routine du quotidien. Mais la réalité lui est hostile (tout comme le climat des bords du Lac Léman) : les événements narratifs lui offrent le choix entre mariage de convenance et amour romantique, impossible à vivre au quotidien. De plus, le narrateur extradiégétique fait commettre à Edith erreur sur erreur, faux pas sur faux pas. Lorsqu'elle avait décidé de quitter David pour épouser Geoffrey Long, elle s'était dit : «Il 
est grand temps que je renonce à mes illusions, les illusions avec lesquelles je suis née, et que je regarde la réalité en face » (HL 127). Elle succombe à nouveau à la tentation de la sécurité d'une position sociale et décide d'accepter le mariage de convenance dépourvu d'amour que lui offre M. Neville. Elle se persuade alors que "l'expérience l'avait mûrie » (HL 188). Mais l'exil n'a pas eu l'effet escompté - Edith n'a rien oublié et son malaise s'est accentué. Mais lorsqu'elle sort de l'hôtel au petit matin pour poster sa lettre d'adieu à David, elle aperçoit M. Neville quittant subrepticement la chambre de Jennifer Pusey. Le roman se clôt sur ce revirement ironique - Edith déchire la lettre d'adieu et envoie un télégramme à David : "elle barra les mots 'Je rentre chez moi' pour écrire simplement 'Je reviens' ", mot sur lequel se clôture le roman (HL 199).

En renonçant à son "chez soi », elle renonce à cet " entourage, qui était devenu soudain inamical», et qui l'avait poussée à être "raisonnable » en optant pour un mariage de convenance. Elle renonce par là-même à une personnalité qu'elle s'était forgée. Comme l'explique Brookner dans une interview : "Je rentre chez moi' impliquerait le retour à une vie de famille bien ordonnée : 'chezsoi' sous-entend un mari, des enfants, une vie rangée, des repas réguliers, 'je reviens' par contre est sa vision plus honnête de la situation. Sur ce point du moins, elle accède effectivement à plus de lucidité ${ }^{10}$. L'exil d'Edith a eu un effet contraire à celui qu'espéraient ses proches : elle choisit (cette fois en toute lucidité), l'Amour désintéressé - Brookner a bien dit qu'elle voulait écrire « une histoire d'amour [...] dans laquelle l'amour triomphait vraiment "11. Mais en restant fidèle à cet homme marié, qui ne lui offre pas de

10 «'Coming home' would be coming back to domestic propriety : 'home' implies husband, children, order, regular meals, but 'Returning' is her more honest view of the situation. To that extent she does break through to a clearer vision. " (John Haffenden, Novelists in Interview, Londres, Methuen, 1985, p. 71).

11 Olga KENYON, Women Writers Talk, Oxford, Lennard Publishing, 1989, p. 13 
«chez-elle », Edith renonce aussi à un foyer - chose qu'elle désire le plus au monde - et choisit la solitude.

Que faut-il en conclure ? S'agit-il d'une fin heureuse ou malheureuse ? D'une victoire ou d'une défaite d'Edith ? D'une régression ou d'une résurrection? Les critiques sont divisés. Nous pensons que la technique narrative peut aider à interpréter cette fin, dont l'ambiguïté reflète les dualités et les discordances qui ne cessent de se faire sentir tout au long du roman. La confusion des voix narratives et des points de vues sert à masquer le fait que le narrateur n'en reste pas moins ironiquement dissocié de la conscience dont il rapporte les mouvements. Les analepses et le récit ouroboros, qui se love sur lui-même, semblent piéger Edith dans un passé dont elle ne saurait se défaire. Les erreurs constantes de jugement de l'héroïne sont ironiquement opposées aux points de vue de personnages plus lucides, ainsi qu'à une réalité narrative inéluctable et contraire à ses espoirs. Le grand amour qui lui est offert n'est ironiquement qu'une aventure avec un homme marié. Edith semble être la victime aveugle d'un ironiste cruel qui ne lui offre jamais la possibilité du bonheur. En choisissant finalement l'Amour, Edith ne succombe-t-elle pas justement à ces mêmes illusions romantiques dont elle nourrit ses lectrices et dont elle se croyait débarrassée ? Pourquoi celle qui se présente elle-même (mais n'en croit pas un mot !) comme stupide - «Je suis plutôt idiote, $j$ 'en ai peur» (HL 100) - tout au long du roman deviendrait-elle subitement lucide?

Le manque d'amour de sa mère, Rosa, dont elle souffrira toute sa vie, a légué solitude et silence à Edith, le déterminisme familial écrasant le libre arbitre. Son malaise initial n'a fait que croître tout au long du roman. Sous l'apparence trompeuse d'une femme raisonnable et maîtresse d'elle-même, par delà une écriture traditionnelle qui prône en apparence la raison, se dessine un autre texte, qui mine et dément le premier. Le style limpide de Brookner est en trompe-l'œil, tout comme son héroïne, et même son narrateur qui se confond avec son personnage, semblant ainsi s'auto-analyser, s'autocritiquer. Puisque l'héroïne, elle-même écrivain, est peu 
crédible - « unreliable » - le narrateur lui-même serait peu crédible. Dans le décalage entre rêve et réalité, entre espoir et lucidité, entre raison et passion, entre le contrôlable et l'incontrôlable, s'insinuent le silence, le vide, l'irréalité d'une vie non vécue, contre lesquels David ne pourra rien. Imaginer Edith heureuse serait se laisser prendre par ces illusions romanesques que vend l'héroöne. Ecoutons plutôt la voix du narrateur, qui à travers la fréquence obsessionnelle des mots de la même famille sémantique que "solitude » et "silence ", laisse entendre que c'est bien à la solitude et au silence qu'Edith est destinée depuis son enfance ${ }^{12}$.

$12 \mathrm{~J}$ 'aimerais ajouter - en post-scriptum, ou «dans une autre voix »-que, dans cette analyse, je me suis cantonnée à la scène énonciative, sans poser la question de l'auteur, ni du hors-texte. Effectivement, cela ne relève pas de la poétique. Néanmoins, je pense qu'il faut aller plus loin dans la recherche du sens du texte. Pour comprendre l'origine de cette double voix narrative, de cette cruauté du narrateur envers son personnage (qui est, en fait cruauté envers soimême, puisque narrateur et personnage se superposent), de cette remise en cause du statut même du narrateur, je pense qu'il faut «aller chercher " l'auteur, car Hotel du Lac est typique de tous les romans très répétitifs de Brookner, qui sont «une sorte de fiction autobiographique ", derrière laquelle on "sent l'auteur". Mais ceci est un autre champ d'investigation. 